\title{
Impact of Herobic Exercise on Restoration of Soleus and Gastrocnemius Muscles Microcirculations in Wistar Rats with Chronic Heart Failure
}

Farzad Nazem (PhD)

Department of Sport Physiology, Faculty of Physical Education and Sport Sciences, Bu-Ali Sina University, Hamedan, Iran

Malihe Ardakani(MSc)

Department of Sport Physiology, Faculty of Physical Education and Sport Sciences, Bu-Ali Sina University, Hamedan, Iran

\section{Mohammad Reza}

Gholami(PhD)

Department of Anatomy, Razi Herbal Medicine Research Center, Lorestan University of Medical Sciences, Khorramabad, Iran

Kamal Ranjbar (PhD)

Department of Physical Education and Sport Sciences, Bandar Abbas Branch, Islamic Azad University, Bandar Abbas, Iran

Afshin Nazari (PhD)

Department of Physiology, Razi Herbal Medicine Research Center, Lorestan University of Medical Sciences, Khoramabad, Iran

Corresponding author: Farzad Nazem

Tel: (+98) 09181117911

E-mail:

Farzadnazem1@yahoo.com

Address: Faculty of Physical Education and Sport Sciences, BuAli Sina University, Hamedan, Iran

Received : 20 Aug 2014

Revised: 08 Oct 2014

Accepted: 02 Oct 2014

\section{ABSTRACT}

Background and Objective: Scientific evidence reveals that the density of skeletal muscle microcirculations decreases in the patients with chronic heart failure. Therefore, this study aimed to determine the impact of submaximal aerobic exercise training on restoration of fast/slow-twitch muscle fibers microcirculation in rats with myocardial infarction.

Methods: In this experimental study, 30 male infarcted Wistar rats were randomly divided into three groups of sham $(\mathrm{N}=10)$, control $(\mathrm{N}=10)$ and exercise $(\mathrm{N}=10)$, four weeks after surgery (blockage of anterior descending artery). Daily aerobic exercise included running on a treadmill for 10 weeks (5 times a week) at a speed of $17 \mathrm{~m} / \mathrm{min}$ within 10 to 50 minutes. The animals were anesthetized and then sacrificed with chloroform, 40 hours after the last training session. The microcirculation density of soleus (S0L) and gastrocnemius (Gw) muscles was evaluated by immunohistochemistry assay.

Results: Capillary density and capillary to SOL muscle fiber ratio significantly increased in response to exercise training. However, capillary density, capillary to Gw muscle fiber ratio and Gw muscle arteriolar density of Wistar rats with chronic heart failure were not significantly affected by the exercise program. Moreover, arteriolar density of Gw muscle increased significantly after the exercise program.

Conclusion: The ten-week aerobic exercise might non-uniformly improve the SOL and Gw muscles microcirculation in rats with chronic heart failure by increasing capillary density and capillary to fiber ratio in the SOL muscle and increasing the arteriolar density of the Gw muscle.

Keywords: Heart Failure, Angiogenesis, Exercise.

This paper should be cited as: Nazem F, Ardakani M, Gholami MR, Ranjbark, Nazari A[Impact of Aerobic Exercise on Restoration of Soleus and Gastrocnemius Muscles Microcirculations in Wistar Rats with Chronic Heart Failure] (2011-14) ] mljgoums. 2016; 10(5):33-39 


\section{INTRODUCTION}

Scientific evidence suggests that heart failure not only affects myocardial function, it reduces the local blood flow and leads to loss of skeletal muscle function. The fatigue caused by physical activity and reduced functional capacity that are common in patients with heart failure could be due to blood flow deficiency and impaired skeletal muscle physiology (1, 2). Studies reveal that the structural and functional characteristics of skeletal muscles change dramatically in response to myocardial infarction (MI). In this regard, laboratory studies indicate muscle mass loss (3), increased number of fast-twitch fibers,decreased slow-twitch fibers (4), and restriction of skeletal muscle blood flow in humans (5) and animals $(6,7)$ after MI. In addition, systemic blood flow in the skeletal muscle of mice with heart failure decreases at rest and during physical activity (8). Experimental studies at the capillary level show that capillary density parameters (9) and capillary/fiber ratio (CF ratio) decrease following MI. On the other hand, at the arteriole level, the arteriole tonic sympathetic vasoconstriction activity increases that decreases the arteriolar diameter and elasticity capacity (10). However, the hypoperfusion phenomenon also further accelerates catabolism, reduces oxidative stress in muscles, decreases intracellular $\mathrm{pH}$, increases production of free radicals and inflammatory cytokines, and leads to cell apoptosis. Meanwhile, chronic hypoxia caused by low systemic blood flow also cause damage to the muscle fibers and eventually leads to atrophy (11). In contrast, endurance exercise training contribute in revascularization of the damaged skeletal muscle through the processes of angiogenesis and arteriolar genesis. Scientific evidence reveals that exercise training significantly increases the number of capillaries in active skeletal muscles. In addition, long-term aerobic exercise mainly influences the endings of the vascular network (capillaries).
When performing physical activity, a set of factors take place including the skeletal muscle ischemic and hypoxic conditions, increase in blood flow or shear stress, vasodilation caused by increased shear stress, mechanical stretch in tissue, skeletal muscle contraction and the resulting metabolites that provide the conditions for vascularization of skeletal muscle tissue. In this regard, experimental studies indicate changes in the skeletal muscle capillary network that depends on the contraction pattern (isotonic or isometric), the type of exercise (endurance, strength or concurrent), work intensity or duration of activity (12). Although the mechanism of vascular repair in the skeletal muscle is not clear, recent studies show that vascularization of the target tissue depends on the myofibrils' type and pattern of using skeletal muscle fibers involved in physical activity (13). Based on the available scientific evidence, this study aimed to determine whether long-term aerobic exercise intervention program affect capillary density and arteriolar density of selected soleus (SOL) and gastrocnemius muscles of rats after $\mathrm{MI}$ and in laboratory conditions. Moreover, we aimed to investigate the possible rehabilitating role of aerobic exercise pattern on microcirculation density of slow/fast-twitch SOL and gastrocnemius muscle fibers in infarcted rats.

\section{MATERIAL AND METHODS}

This experimental-laboratory study was performed on 30 male Wistar rats aged 6-8 weeks with a weight range of 180-210 g. Day/night light cycle regulation was done in standard laboratory environment (12-hour light/12-hour dark that began at 8 am). The animals had free access to food and water. Laboratory temperature was stabilized at $24 \pm$ $2{ }^{\circ} \mathrm{C}$ with $46-51 \%$ humidity. The subjects were divided into groups of four, and kept in standard polypropylene cages. The subjects with MI were randomly divided into a control $(\mathrm{N}=10$, Sed-MI) and an exercise intervention 
group $(\mathrm{N}=10, \mathrm{Ex}-\mathrm{MI})$, four weeks after surgery.In addition, sham group $(\mathrm{N}=10)$ included opened-chest rats with no $\mathrm{MI}$. Characteristics of the subjects are presented in Table 1.

First, rats were anesthetized with intraperitoneal injection of thiopental sodium (50 $\mathrm{mg} / \mathrm{kg}$ ip). They were placed supine, their chest area was shaved and then they were placed on the operating table.The rats were prepared for intubation after complete anesthetization. Subject were connected to a ventilator (Model 683 Small Animal Ventilator, Harvard-USA) specific for rodents.Thoracic cavity was opened gently to expose the heart.Then, left anterior descending artery (LAD) was blocked with 0.6 silk threads. Lead II ECG (Powerlab) was recorded to ensure induction of MI. For the sham group, silk threads were passed underneath the LAD.After open surgery, the animals were exposed to pure oxygen to gradually recover from anesthesia.

Four weeks after surgery, rats in the exercise group were subjected to an exercise program consisting of running on treadmill for
rodents.This group performed long-term aerobic activity continuously with an increasing intensity for 10 weeks. The subjects performed running exercise at $10 \mathrm{~m} / \mathrm{min}$ for 10 min during the first week.In the second week, they were forced to run at $14 \mathrm{~m} / \mathrm{min}$ for 20 minutes. In the third week, the subjects performed the activities at $17 \mathrm{~m} / \mathrm{min}(1 \mathrm{~km} / \mathrm{h})$ for 30 minutes. This group run at a constant speed of $17 \mathrm{~m} / \mathrm{min}$ [equivalent to $50-60 \%$ of maximal oxygen consumption $\left(\mathrm{VO}_{2}\right.$ max)], with increase in the duration of the activity in each workout from 40 to 50 minutes. However, the intensity and duration of aerobic exercise remained constant from the fifth week until the tenth week.The rodent treadmill's incline was set at zero during the first three weeks, and then increased to $5 \%$ in the fourth week. However, the incline remained stable at $10 \%$ during weeks 5 to 10 (Table 2) (14). The rats performed treadmill exercise voluntarily. When the animal was feeling fatigued, treadmill was stopped for a short time (less than a minute).Meanwhile, the control (infarcted) and sham groups performed no exercise during the aerobic exercise program.

Table 1- General characteristics of subjects in the experimental groups

\begin{tabular}{|c|c|c|c|}
\hline Group & Sham & Sed-MI & Ex-MI \\
\hline Number & 10 & 10 & 10 \\
\hline Age (Weeks) & 6-8 & 6-8 & $6-8$ \\
\hline Wight of Body (g) & $336 \pm 23$ & $356 \pm 41$ & $321 \pm 19$ \\
\hline Wight of Soleus (mg) & $117 \pm 18$ & $121 \pm 23$ & $125 \pm 32$ \\
\hline Wight of Gastrocnemius (mg) & $1232 \pm 230$ & $1200 \pm 176$ & $1364 \pm 241$ \\
\hline
\end{tabular}

Table 2-Exercise training program

\begin{tabular}{lcccccccccc}
\hline \multicolumn{1}{c}{ Weeks } & $\mathbf{1}$ & $\mathbf{2}$ & $\mathbf{3}$ & $\mathbf{4}$ & $\mathbf{5}$ & $\mathbf{6}$ & $\mathbf{7}$ & $\mathbf{8}$ & $\mathbf{9}$ & $\mathbf{1 0}$ \\
& & & & & & & & & & \\
\hline Intensity (m/min) & $\mathbf{1 0}$ & $\mathbf{1 4}$ & $\mathbf{1 7}$ & $\mathbf{1 7}$ & $\mathbf{1 7}$ & $\mathbf{1 7}$ & $\mathbf{1 7}$ & $\mathbf{1 7}$ & $\mathbf{1 7}$ & $\mathbf{1 7}$ \\
Time (min) & $\mathbf{1 0}$ & $\mathbf{2 0}$ & $\mathbf{3 0}$ & $\mathbf{4 0}$ & $\mathbf{5 0}$ & $\mathbf{5 0}$ & $\mathbf{5 0}$ & $\mathbf{5 0}$ & $\mathbf{5 0}$ & $\mathbf{5 0}$ \\
Incline (\%) & $\mathbf{0}$ & $\mathbf{0}$ & $\mathbf{0}$ & $\mathbf{5}$ & $\mathbf{1 0}$ & $\mathbf{1 0}$ & $\mathbf{1 0}$ & $\mathbf{1 0}$ & $\mathbf{1 0}$ & $\mathbf{1 0}$ \\
\hline
\end{tabular}


The subjects were sacrificed 72 hours after the last session of aerobic exercise, in desiccator containing a cotton wool soaked in chloroform. Then, middle section of the soleus (SOL) muscle and superficial, white part of the gastrocnemius $(\mathrm{Gw})$ muscle of the animal's right leg were removed. Tissue samples were kept in paraffin at $-20 \quad{ }^{\circ} \mathrm{C}$ for immunohistochemistry testing.After cutting 5micron thick sections and preparation of slides for determination of capillary density and CF ratio, hematoxylin and eosin staining was performed.Arteriolar density was evaluated using alpha-actin antibodies (SantaCruz Biotechnology, Santa Cruz, CA, USA). Then, the slides were observed and studied using an Olympus microscope (BX53, Shinjuku, Tokyo, Japan) and the ImageJ software. In order to measure the desired indices (capillary density, CF ratio and arteriolar density), arterioles, muscle fibers and arteries were counted on 10 random microscope fields per section (200x magnification) and expressed per square millimeter. The normality of data distribution was confirmed by the Kolmogorov-Smirnov test. One-way analysis of variance (ANOVA) and Tukey's test were used at significance level of $\mathrm{P}<0.05$. Data were reported as mean $\pm \mathrm{SEM}$.

\section{RESULTS}

There was significant difference in the capillary density of SOL muscle between the groups $(\mathrm{P}=0.003, \mathrm{~F}=9.42)$. In contrast, the capillary density of the control group reduced significantly compared to that of sham group $(\mathrm{P}=0.03)$. The 10-week aerobic exercise increased the capillary density of SOL muscle compared to control group ( $\mathrm{P}=0.025)$, but that is not the case for sham group $(\mathrm{P}=0.69)$. The capillary density of the Gw muscle differed significantly between the groups $(\mathrm{P}=0.04$, $\mathrm{F}=6.01)$. Gw muscle capillary density in the infarcted groups reduced significantly compared to that of the sham group $(\mathrm{P}<0.05)$. However, endurance training increased the $\mathrm{Gw}$ muscle capillary density by $8 \%$ in comparison with control group ( $\mathrm{p}=0.056)$. The capillary density of $\mathrm{Gw}$ muscle in the exercise group was significantly lower than that of in the sham group $(\mathrm{P}=0.01)$. Moreover, the $\mathrm{CF}$ ratio in SOL muscle increased significantly in response to aerobic exercise compared to control group $(\mathrm{P}=0.01)$. However, such difference was not observed in the case of SOL muscle from the sham group and CF ratio of the $\mathrm{Gw}$ muscle.On the other hand, the immunohistochemical evaluation revealed that the arteriolar density of SOL muscle in different groups had no significant difference $(\mathrm{P}=0.7, \mathrm{~F}=2.04)$. Arteriolar density of the $\mathrm{Gw}$ muscle in the control and sham groups had no significant difference.However, 10 weeks of exercise significantly increased the $\mathrm{Gw}$ muscle's arteriolar density in comparison with the sham and control groups (Table 3).

Table 3- Revascularization of SOL and Gwmuscles

\begin{tabular}{|c|c|c|c|c|c|c|}
\hline & & Lmuscle & & & muscle & \\
\hline Group & $\begin{array}{c}\text { Capillary } \\
\text { density }\end{array}$ & CF ratio & $\begin{array}{c}\text { Arteriolar } \\
\text { density }\end{array}$ & $\begin{array}{c}\text { Capillary } \\
\text { density }\end{array}$ & CF ratio & $\begin{array}{c}\text { Arteriolar } \\
\text { density }\end{array}$ \\
\hline Sham & $813 \pm 23$ & $2.2 \pm 0.6$ & $65 \pm 5$ & $567 \pm 29$ & $1.2 \pm 0.1$ & $21 \pm 5$ \\
\hline Sed-MI & $600 \pm 17 *$ & $1.6 \pm 0.3 *$ & $69 \pm 10$ & $465 \pm * 30$ & $1.1 \pm 0.1$ & $17 \pm 4$ \\
\hline Ex-MI & $772 \pm 36 \&$ & $2.0 \pm 0.5 \&$ & $77 \pm 18$ & $504 \pm 16$ & $1.5 \pm 0.3$ & $28 \pm 11 \& *$ \\
\hline
\end{tabular}

Data are represented as mean \pm SEM; * $P<0.05$ vs. sham group; $P<0.05$ vs. sed group 


\section{DISCUSSION}

Based on the findings, the capillary density of SOL and Gw muscles in four-week infarcted rats reduced significantly by $26 \%$ and $17 \%$, respectively. However, the arteriolar density of the muscles did not change significantly after MI. This is consistent with the results of Ogoh et al. study that reported a significant reduction in the capillary density of slow-twitch SOL muscle fibers and fast-twitch long toe extensor muscle fibers four weeks after MI, by $18.5 \%$ and $18.2 \%$, respectively (15). Unlike the capillary density, 4-week post-MI arteriolar density of the SOL and Gw muscles did not change significantly.

No scientific evidence was found on arteriolar changes of the skeletal muscles after heart failure. Our study also showed that 10 weeks of submaximal aerobic training at 50-60\% $\mathrm{VO}_{2}$ max four week after MI, helped restore capillary density and capillary to SOL muscle fiber ratio, but did not affect the arteriolar density of this muscle.This is consistent with the findings of Suzoki et al. that showed SOL muscle capillary density in healthy rats does not change significantly in response to endurance treadmill running at a speed of 20 $\mathrm{m} / \mathrm{min}$ (16). This inconsistency could be due to the differences in parameters related to health and sickness of animals (MI) or physiological adaptation of the target tissue in response to aerobic exercise training. In this regard, study of White et al. suggested that the changes in blood vessel density depend on length of the exercise program (13). On the other hand, longterm aerobic exercise in the present study significantly increased the arteriolar density of gastrocnemius muscle compared to sham and control groups. Consistent with the findings of the present study, Laughlin et al. noted that 10 weeks of aerobic exercise with intensity of 30 $\mathrm{m} / \mathrm{min}$ significantly increases the gastrocnemius muscle arteriolar density of healthy rats, but does not affect their SOL muscle arteriolar density (17).

Considering these findings, this study seems to be the first to evaluate the effect of submaximal aerobic exercise (running) on reconstruction of blood vessels in skeletal muscles of rats with heart failure. Given the limited number of available studies, the mechanism of changes in blood vessels of fast/slow-twitch muscle fibers after heart failure is unclear. On the other hand, the results of this study show that regeneration of blood vessels in the fast/slow-twitch skeletal muscle fibers following chronic aerobic exercise does not follow the same pattern. It seems that this type of activity mostly affects the capillary density, capillary to SOL muscle fibers ratio and gastrocnemius muscle arteriolar density.In general, characteristics of skeletal muscle vascularization depend on muscle fibers recruitment pattern, composition of muscle fibers and secretion of factors involved in angiogenesis. Study of Gute et al. also showed that the highest rate of capillary growth in response to exercise was seen in muscles with the greatest relative increase in contractile activity (18). On the other hand, angiogenesis and arteriolargenesis were triggered in response to exercise training by local metabolic factors (such as adenosine)induced by muscle fiber recruitment and shear stress, which led to secretion of angiogenic factors (19). Difference in CF ratio of various muscle fibers (20) or dependence of skeletal muscle angiogenic response to exercise on the type of muscle fiber (18) indicated that the signals required to initiate the angiogenesis process, originated in the contacted muscle fibers. In this regard, study of Brutsaert et al. demonstrated that secretion of vascular endothelial growth factor (VEGF) was the most important mitogen for endothelial cells that played a key role in angiogenesis, so that the role of oxidative fibers (slow-twitch) was more than the fasttwitch glycolytic fibers, following a session of treadmill exercise (21).Since submaximal aerobic exercise mostly recruits slow-twitch muscle fibers, it is likely that the increased VEGF secretion and presence of angiogenic metabolites such as adenosine lead to more increase in the capillary density of slow- 
twitch muscle fibers compared to fast-twitch muscle fibers. Thus, local differences in the skeletal muscle capillary density between humans and animals with chronic heart failure in response to exercise training could be due to the unique phenotypic characteristics of muscle fibers. In the present study, submaximal endurance exercise increased the SOL and gastrocnemius muscles arteriole densities by $10 \%$ and $39 \%$, respectively. Increased arteriolar density and unaltered capillary density of the gastrocnemius muscle in the exercise group indicate the hypothesis that the fast-twitch muscle fibers experience arteriole argenesis more than the slow-twitch muscle fibers. In this regard, the previous studies showed increased arteriolar density following exercise, induced by increase in density of arterioles with diameter of 8 to 20 micrometers (22).In fact, the formation of new arteries caused by the migration of smooth muscle cells to the capillary wall ultimately leads to generation of arterioles from capillaries (arteriolargenesis).In this regard, reports indicate the role of exercise training in stimulation of the arteriolargenesis process with an unknown mechanism (13). However, the balance between angiogenic factors such as tumor growth factor that leads to the proliferation and migration of smooth muscle endothelial cells (23) and recruitment of pericytes (24) is controlled by angiostatic factors such as angiostatin that inhibits the mentioned processes $(25,26)$. Thus, it is necessary to evaluate the contribution of each of these factors in various muscle fibers in response to exercise training to gain a better understanding of changes in the arteriolargenesis process in different types of muscle fibers. Muscle tissue's partial pressure of oxygen $\left(\mathrm{PO}_{2}\right)$ is another factor affecting the arteriolargenesis process during exercise (27). The oxidative slow-twitch muscle fibers have more capillary density. Therefore, they might experience less $\mathrm{PO}_{2}$ decline during endurance training compared to fast-twitch muscle fibers. These changes will eventually lead to stimulation of arteriolargenesis in the fasttwitch muscle fibers.

\section{CONCLUSION}

The results reveal that long-term submaximal aerobic exercise intervention at $50-60 \% \mathrm{VO}_{2}$ max in infarcted rats might be effective in restoring SOL muscle capillary density and increasing the gastrocnemius muscle arteriolar density. In other words, aerobic exercise at intensities below the lactate threshold could act as an agent for cardiac rehabilitation of rats with chronic MI.

\section{ACKNOWLEDGEMENTS}

This project was funded by the Department of Research and Technology of Bu-Ali Sina University. The authors acknowledge the research authorities and staff of Medicinal Plant Research Center laboratory at Lorestan University of Medical Sciences.

\section{CONFLICT OF INTEREST}

The authors declare no conflicts of interest regarding this manuscript. 


\section{REFERENCES}

1. Katz SD, Yuen J, Bijou R, Lejemtel TH. Training improves endothelium-dependent vasodilation in resistance vessels of patients with heart failure. Journal of Applied Physiology. 1997; 82(5): 1488-92.

2. Hornig B, Maier V, Drexler H. Physical training improves endothelial function in patients with chronic heart failure. Circulation. 1996; 93(2): 210-4.

3. Magnusson G, Kaijser L, Rong H, Isberg B, Sylven C, Saltin B. Exercise capacity in heart failure patients: relative importance of heart and skeletal muscle. Clinical Physiology. 1996; 16(2): 183-95.

4. Sullivan MJ, Green HJ, Cobb FR. Skeletal muscle biochemistry and histology in ambulatory patients with long-term heart failure. Circulation. 1990; 81(2): 518-27. 5. Drexler H, Riede U, Münzel T, König H, Funke E, Just $\mathrm{H}$. Alterations of skeletal muscle in chronic heart failure. Circulation. 1992; 85(5): 1751-9.

6. Didion SP, Carmines PK, Ikenaga H, Mayhan WG. Enhanced constrictor responses of skeletal muscle arterioles during chronic myocardial infarction. American Journal of Physiology-Heart and Circulatory Physiology. 1997; 273(3): H1502-H8.

7. Thomas DP, Hudlicka O, Brown MD, Deveci D. Alterations in small arterioles precede changes in limb skeletal muscle after myocardial infarction. American Journal of Physiology-Heart and Circulatory Physiology. 1998; 275(3): H1032-H9.

8. Kindig CA, Musch TI, Basaraba RJ, Poole DC. Impaired capillary hemodynamics in skeletal muscle of rats in chronic heart failure. Journal of Applied Physiology. 1999; 87(2): 652-60.

9. Schieffer B ,Wollert K, Berchtold M, Saal K, Schieffer $\mathrm{E}$, Hornig $\mathrm{B}$, et al. Development and prevention of skeletal muscle structural alterations after experimental myocardial infarction. American Journal of PhysiologyHeart and Circulatory Physiology. 1995; 269(5): H1507H13.

10. Thomas DP, Hudlická O. Arteriolar reactivity and capillarization in chronically stimulated rat limb skeletal muscle post-MI. Journal of Applied Physiology. 1999; 87(6): 2259-65.

11. Dobsak P, Nováková M, Siegelová J, Fiser B, Vitovec J, Nagasaka M ,et al. Low-frequency electrical stimulation increases muscle strength and improves blood supply in patients with chronic heart failure. Circulation Journal. 2006; 70(1): 75-82.

12. Prior BM, Yang H, Terjung RL. What makes vessels grow with exercise training? Journal of Applied Physiology. 2004; 97(3): 1119-28.

13. White FC, Bloor CM, McKirnan MD, Carroll SM. Exercise training in swine promotes growth of arteriolar bed and capillary angiogenesis in heart. Journal of Applied Physiology. 1998; 85(3): 1160-8.

14. Leosco D, Rengo G, Iaccarino G, Golino L, Marchese M, Fortunato F, et al. Exercise promotes angiogenesis and improves $\beta$-adrenergic receptor signalling in the post-ischaemic failing rat heart. Cardiovasc Res. 2008 May 1;78(2):385-94.
15. Ogoh S, Hirai T, Nohara R, Taguchi S. Adaptation in properties of skeletal muscle to coronary artery occlusion/reperfusion in rats. Nihon Seirigaku Zasshi. 2002; 64(10): 225-36.

16. Suzuki J. L-arginine supplementation causes additional effects on exercise-induced angiogenesis and $V E G F$ expression in the heart and hind-leg muscles of middle-aged rats. The Journal of Physiological Sciences. 2006; 56(1): 39-44.

17. Laughlin $\mathrm{MH}$, Cook JD, Tremble R, Ingram $\mathrm{D}$, Colleran PN, Turk JR. Exercise training produces nonuniform increases in arteriolar density of rat soleus and gastrocnemius muscle. Microcirculation. 2006; 13(3): 175-86.

18. Gute D, Laughlin MH, Amann JF. Regional changes in capillary supply in skeletal muscle of interval-sprint and low-intensity, endurance-trained rats. Microcirculation. 1994; 1(3): 183-93.

19. Deveci D, Marshall J, Egginton S. Chronic hypoxia induces prolonged angiogenesis in skeletal muscles of rat. Experimental physiology. 2002; 87(03): 287-91.

20. Waters RE, Rotevatn S, Li P, Annex BH, Yan Z. Voluntary running induces fiber type-specific angiogenesis in mouse skeletal muscle. American Journal of Physiology-Cell Physiology. 2004; 287(5): C1342-C8.

21. Brutsaert TD, Gavin TP, Fu Z, Breen EC, Tang K, Mathieu-Costello $\mathrm{O}$, et al. Regional differences in expression of VEGF $m R N A$ in rat gastrocnemius following $1 \mathrm{hr}$ exercise or electrical stimulation. BMC physiology. 2002; 2(1): 8 .

22. Laughlin M, Roseguini B. Mechanisms for exercise training-induced increases in skeletal muscle blood flow capacity: differences with interval sprint training versus aerobic endurance training. Journal of physiology and pharmacology: an official journal of the Polish Physiological Society. 2008; 59(Suppl 7): 71.

23. Tsai S, Hollenbeck ST, Ryer EJ, Edlin R , Yamanouchi D, Kundi R, et al. TGF- $\beta$ through Smad3 signaling stimulates vascular smooth muscle cell proliferation and neointimal formation. American Journal of Physiology-Heart and Circulatory Physiology. 2009; 297(2): H540-H9.

24. Chantrain CF, Henriet $P$, Jodele $S$, Emonard H, Feron $\mathrm{O}$, Courtoy PJ, et al. Mechanisms of pericyte recruitment in tumour angiogenesis: a new role for metalloproteinases. European Journal of Cancer. 2006; 42(3): 310-8.

25. Nyberg P, Xie L, Kalluri R. Endogenous inhibitors of angiogenesis. Cancer research. 2005; 65(10): 3967-79.

26. Walter JJ, Sane DC. Angiostatin binds to smooth muscle cells in the coronary artery and inhibits smooth muscle cell proliferation and migration in vitro. Arteriosclerosis, thrombosis, and vascular biology. 1999; 19(9): 2041-8.

27. Suzuki J, Gao M, Batra S, Koyama T. Effects of treadmill training on the arteriolar and venular portions of capillary in soleus muscle of young and middle-aged rats. Acta physiologica scandinavica. 1997; 159(2): 11321. 